Received: 20 April 2018

Accepted: 6 February 2019

Published online: 12 March 2019

ENTIFIC REP

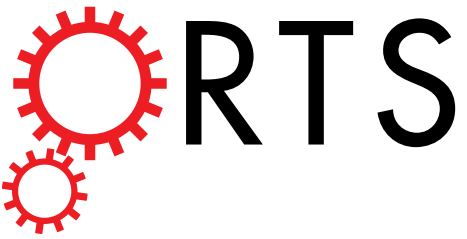

OPEN

\title{
A novel RT-QPCR-based assay for the relative quantification of residue specific m6A RNA methylation
}

\author{
Ainara Castellanos-Rubio ${ }^{1,2,5}$, Izortze Santin ${ }^{3,5}$, Ane Olazagoitia-Garmendia ${ }^{1}$, \\ Irati Romero-Garmendia ${ }^{1}$, Amaia Jauregi-Miguel ${ }^{1}$, Maria Legarda ${ }^{4}$ \& Jose Ramon Bilbao ${ }^{1,5}$
}

N6-methyladenosine ( $\mathrm{m} 6 \mathrm{~A}$ ) is the most common and abundant RNA modification. Recent studies have shown its importance in the regulation of several biological processes, including the immune response, and different approaches have been developed in order to map and quantify m6A marks. However, site specific detection of $\mathrm{m6A}$ methylation has been technically challenging, and existing protocols are long and tedious and often involve next-generation sequencing. Here, we describe a simple RTQPCR based approach for the relative quantification of candidate $\mathrm{m} 6 \mathrm{~A}$ regions that takes advantage of the diminished capacity of Bstl enzyme to retrotranscribe $\mathrm{m6A}$ residues. Using this technique, we have been able to confirm the recently described m6A methylation in the 3'UTR of SOCS1 and SOCS3 transcripts. Moreover, using the method presented here, we have also observed alterations in the relative levels of $\mathrm{m} 6 \mathrm{~A}$ in specific motifs of SOCS genes in celiac disease patients and in pancreatic $\beta$-cells exposed to inflammatory stimuli.

N6-methyladenosine (m6A) is the most abundant internal modification on mammalian mRNAs and long-noncoding RNAs. These modifications are generally dynamic and exhibit changes in distribution or frequency in response to certain stimuli, constituting a new layer of gene regulation termed epitranscriptomics ${ }^{1,2}$. Genetic loss-of-function studies for m6A methyltransferases (m6A writers), m6A-binding proteins (m6A readers), and m6A demethylases (m6A erasers) have highlighted a critical role for m6A modifications in the control of gene expression in a range of physiological processes ${ }^{3,4}$. A recent study analyzing the effects of deleting writer Mettl 3 in mouse T-cells found that, in the absence of Mettl3, mice develop a chronic inflammation of the intestine and that the mRNAs for STAT signaling inhibitory proteins SOCS1 and SOCS3, that generally present m6A methylation marks in their $3^{\prime}$ UTR region, exhibited slower decay, resulting in increased mRNA and protein levels, and establishing a link between the m6A methylation of those transcripts and T-cell responses ${ }^{5,6}$. More recently, m6A has been shown to affect the LPS-induced inflammatory response in human dental pulp cells ${ }^{7}$ opening a new layer of investigation into the molecular mechanisms of inflammatory diseases.

Technological advances in high-throughput sequencing in combination with antibody-enrichment and/ or induced nucleotide-specific chemical modifications have accelerated the mapping of epitranscriptomic modifications. However, site-specific detection and quantification of $\mathrm{m} 6 \mathrm{~A}$ has been technically challenging $^{8}$. Methods developed so far involve long and tedious procedures like SCARLET (site-specific cleavage and radioactive-labeling followed by ligation-assisted extraction and thin-layer chromatography $)^{9}$ and miCLIP (m6A individual-nucleotide-resolution cross-linking and immunoprecipitation) ${ }^{10}$ or are based on the diminished capacity of different nucleic acid polymerases, namely Tth (Thermus thermophiles) and BstI (Bacillus

${ }^{1}$ Department of Genetics, Physical Anthropology and Animal Physiology, University of the Basque Country (UPVEHU), Biocruces-Bizkaia Health Research Institute, Leioa, Spain. ${ }^{2}$ IKERBASOUE, Basque Foundation for Science, Bilbao, Spain. ${ }^{3}$ Department of Biochemistry and Molecular Biology, University of the Basque Country (UPV-EHU), Endocrinology and Diabetes Research Group, Biocruces-Bizkaia Health Research Institute, Leioa, Spain. ${ }^{4}$ Pediatric Gastroenterology Unit, Cruces University Hospital, University of the Basque Country (UPV-EHU), Barakaldo, Spain. ${ }^{5}$ Spanish Biomedical Research Center in Diabetes and Associated Metabolic Disorders (CIBERDEM), Madrid, Spain. Correspondence and requests for materials should be addressed to A.C.-R. (email: ainara.castellanos@ehu.eus) or J.R.B. (email: joseramon.bilbao@ehu.eus) 
A

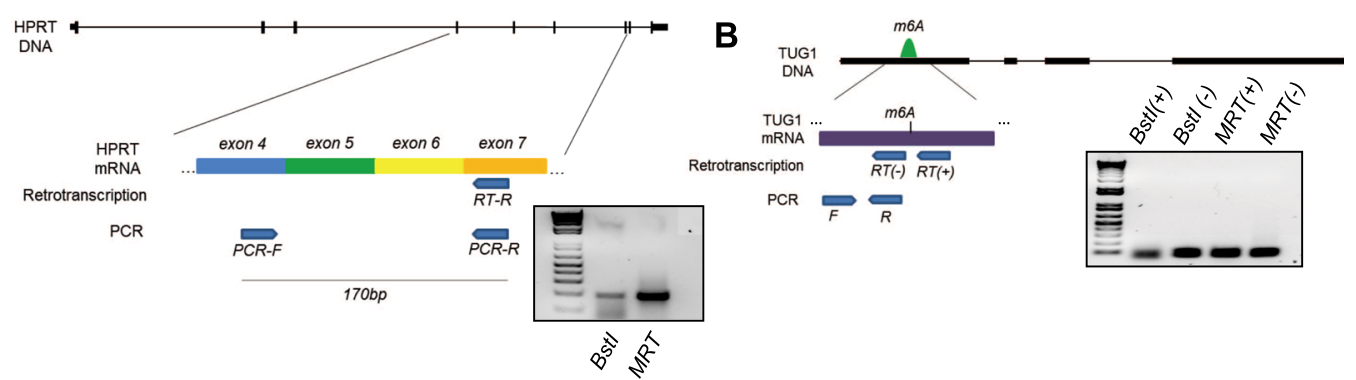

C

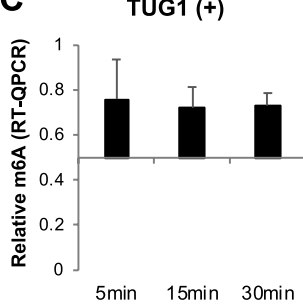

HPRT (-)

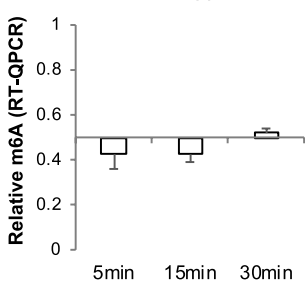

D

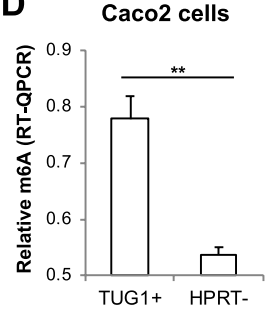

E

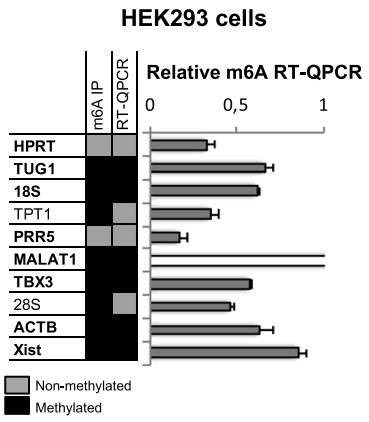

Figure 1. (A) Graphical representation of retrotranscription followed by PCR of HPRT (left). Agarose gel after PCR of the retrotranscribed samples using BstI and MRT enzymes (right). (B) Graphical representation of retrotranscription followed by PCR of TUG1 lncRNA. m6A mark (in green) was described by SCARLET method (Liu, N. et al..$^{9}$ ) (left). Agarose gel after PCR of the retrotranscribed samples using m6A (+) and (-) primers and BstI and MRT enzymes. (C) m6A-RT-QPCR using different time points ( $5 \mathrm{~min}, 15 \mathrm{~min}$ and $30 \mathrm{~min}$ ) for the retrotranscription of methylated TUG1 and unmethylated HPRT mRNAs. RNA was extracted from three different passages of HCT15 intestinal cell line. (D) Relative quantification of m6A levels of TUG1 and HPRT in Caco 2 intestinal cells. Data are represented as the mean and standard error of three independent experiments. $* *$ p-value $<0.01$ based on paired Student's t-test. (E) Comparison of m6A IP data retrieved from the Met-DB v2.0 database and relative m6A RT-QPCR results in different genes using the HEK293 cell line. Genes in bold showed concordant results using both methods. Grey boxes represent negative (non-methylated) and black boxes represent positive (methylated) results. Relative RT-QPCR data correspond to the mean and standard error of four independent experiments.

stearothermophilus), to retrotranscribe m6A nucleotides located adjacent to the priming oligo ${ }^{11,12}$. Flexibility is necessary for the processive movement of the enzyme during retrotranscription and the conformational freedom between the complex formed by the primer, the template and the enzyme itself is decreased in RNA templates that contain $\mathrm{m} 6 \mathrm{~A}$ residues ${ }^{12,13}$. Single-nucleotide primer extension with these enzymes followed by polyacrylamide gel electrophoresis of the extension products has already been used to quantify the m6 methylation levels of specific sites ${ }^{11,12,14}$. In the present study, we have simplified the protocol while improving accuracy in the relative quantification of m6A nucleotides by taking advantage of the inherent reverse transcriptase activity of BstI polymerase, hampered in the presence of $\mathrm{m} 6 \mathrm{~A}$ as previously described by Shi, $\mathrm{C}$ et al. ${ }^{15}$ in combination with relative quantification using PCR. We have used this novel m6A-RT-QPCR approach to quantify the m6A levels in specific residues of human SOCS1 and SOCS3 mRNAs, members of one of the major mechanisms for the regulation of cytokine signaling ${ }^{16}$, in intestinal and $\beta$-pancreatic cell lines and in intestinal biopsy samples. Thus, the aim of this study was to develop a simple and cost-effective method to relatively quantify the m6A levels of candidate transcripts that could contribute to the development of different types of diseases, such as those related to the immune system.

\section{Results}

m6A-induced reduction in Bst/ retrotranscription efficiency can be assessed by QPCR. It has been previously shown that BstI polymerase has an inherent retrotranscriptase (RT) activity ${ }^{12,15}$, but we wanted to evaluate its capacity to retrotranscribe mRNA using dNTPs instead of a single nucleotide. We designed a reverse primer in exon 7 of $H P R T$ gene and performed RT reactions using BstI or $M-M u L V$ RT (MRT) enzymes and RNA extracted from HCT15 intestinal cells (Fig. 1A). To verify the ability of BstI for retrotranscription, we subsequently amplified the retrotranscription reaction products by PCR using a forward primer in exon 4 and a reverse primer in exon 7, which ensures that the product will be derived from mRNA and not from any trace of genomic DNA. Both enzymes are able to synthesize cDNA from an mRNA template but, as expected, $M R T$ outperforms BstI in retrotranscription efficiency (Fig. 1A).

Having confirmed the retrotranscriptase activity of BstI polymerase in our experimental conditions, we wanted to evaluate its ability to reverse transcribe a known m6A residue in the TUG1 lncRNA ${ }^{7}$. We performed 
two RT reactions; one with a primer located adjacent to the test m6A residue, or primer $(+)$, and another one with no m6A nearby, or primer (-) (Fig. 1B). After PCR amplification of the RT product, we could detect differences in band intensities between the two reactions performed with BstI, suggesting a higher RT efficiency for the reaction in which primer (-) was used (Fig. 1B). On the contrary, PCR of the RT products from the MRT reactions suggest that this enzyme is not affected by the proximity of an m6A mark to the primer. In view of these results, we hypothesized that those differences in RT efficiency caused by the presence of m6A could be relatively quantified by a QPCR step following retrotranscription. In order to test this possibility, HPRT gene was used as a negative control for m6A (no methylation marks are described in this transcript), while TUG1 was used as a positive control. The following formula was stablished for the calculation of the relative m6A levels after QPCR:

$$
\text { Relative } m 6 \mathrm{~A}=2^{-(\text {Ctprimer }(-) B s t I-C t p r i m e r(-) M R T) /(C t p r i m e r(+) B s t I-C t p r i m e r(+) M R T)}
$$

Based on these calculations, in the absence of m6A marks, the differences of retrotranscription efficiencies between the two enzymes will be similar regardless of the primer used, and the values of the relative m6A quantification will be close to 0.5 . In the presence of an $\mathrm{m} 6 \mathrm{~A}$ residue, due to the decreased retrotranscription ability of $B s t I$, the difference in RT efficiencies between enzymes will be more pronounced when using the positive $(+)$ primer (adjacent to the $\mathrm{m} 6 \mathrm{~A}$ residue), resulting in higher values $(>0.5)$ of relative m6A quantification.

In order to determine the best time-point for the quantification of $\mathrm{m} 6 \mathrm{~A}$ based on the differences in enzyme performance, we set up a m6A-RT-QPCR time-course for TUG1 and HPRT genes in RNA samples extracted from three different passages of the human HCT15 intestinal cell line. As seen in Fig. 1C, the m6A-positive region in TUG1 mRNA showed positive values (above 0.5) in all three time points. However, the m6A-negative region in the HPRT gene started to show values above the threshold after 30 minutes of retrotranscription, so all subsequent experiments were done after 15 minutes of retrotranscription. Under these conditions, m6A-RT-QPCR analyses confirmed the positive and negative m6A status in TUG1 and HPRT genes, respectively, when using RNA extracted from Caco2 intestinal cell line (Fig. 1D), indicating that this method is useful to relatively quantify the $\mathrm{m} 6 \mathrm{~A}$ methylation at a single nucleotide level. To extend the applicability of the method, we quantified relative m6A methylation on several additional RNA residues and compared the results with previously published m6A immunoprecipitation data of the same regions available at the Met-DB v2.0 database ${ }^{17}$. For this purpose, we used RNA from HEK293 cells as it was the material used in the immunoprecipitation experiments. Eight out of the ten gene regions analyzed by relative m6A RT-QPCR method presented concordant results in terms of methylation status (methylated/non-methylated) when compared with those available in the database (Fig. 1E) confirming the high efficiency of this method.

Fluctuations in m6A levels can be detected using m6A-RT-QPCR. In order to assess the ability of this technique to detect fluctuations in m6A levels, the same TUG1 region was in vitro transcribed (IVT) to generate a single stranded RNA without any methylation. Increasing amounts of non-methylated IVT RNA were mixed with RNA extracted from HCT15 cells and the ability to detect m6A marks was quantified. The methylation levels of TUG1 residue decreased but remained positive after adding 1500 copies of non-methylated transcript and got closer to negative values after adding 150,000 and 1,500,000 copies of the non-methylated RNA (Fig. 2A).

To further confirm the suitability of this method for relative quantification of m6A levels in different RNA transcripts in the HCT15 cell line, we used primers adjacent to other described m6A motifs in ACTB mRNA and $18 S \mathrm{RNA}^{9,12}$. As was the case for TUG1, both transcripts showed methylation in the selected motifs, however, only the levels observed in $18 S$ were statistically significant compared to the non-methylated control HPRT (Fig. 2B). To determine the ability of this method to quantify m6A fluctuation levels we incubated cells with different concentrations of cycloleucine, an inhibitor of m6A methylation ${ }^{18}$, in order to get RNA with decreased methylation percentage. Incubation of cells with $100 \mathrm{mM}$ cycloleucine shows $35 \%$ average reduction in the general $\mathrm{m} 6 \mathrm{~A}$ signal detected by RNA Dot-Blot (Fig. 2C) using an anti-m6A antibody. These results were concordant with the relative quantification by m6A-RT-QPCR of the TUG1 and $18 \mathrm{~S}$ regions, which showed a decreased abundance of m6A of $25 \%$ after cycloleucine treatment (Fig. 2D).

Methylation in SOCS1 and SOCS3 3'UTR can be assessed by m6A-RT-QPCR. In order to further validate our method for the quantification of m6A levels in additional transcripts, we analyzed the methylation levels of SOCS1 and SOCS3 mRNAs. The methylation of these two transcripts has been recently associated with their stability and subsequently with the ability of T-cells to respond to certain stimuli as the proinflammatory cytokine IL7 ${ }^{5}$. As m6A marks in these genes have been described to be located in the $3^{\prime}$ UTR, we analyzed their sequences in order to find the DRACH m6A consensus motif ${ }^{10}$. SOCS1 presents a single consensus motif (GGACC), m6A-256 (Fig. 3A), while several motifs are found in the SOCS3 gene. For the latter, we selected to evaluate one of the two GGACU motifs, m6A-1132, as it has been described to be the most commonly methylated sequence (Fig. 3B). We were able to confirm the methylation of the selected regions in both SOCS1 and SOCS3 in RNA from intestinal origin using RNA immunoprecipitation with an antibody against m6A followed by QPCR (Fig. 3C,D).

We next evaluated the mRNA methylation of SOCS1 and SOCS3 m6A motifs in Caco2 and HCT15 intestinal cell lines using our novel m6A-RT-QPCR method. Both intestinal cell lines showed significantly positive levels of m6A methylation in the SOCS3 motif (Fig. 3E,F). The methylation levels of SOCS1 reached statistical significance only in the HCT15 cells. These results confirm that this method can be used to relatively quantify the m6A methylation level of a single base in different transcripts.

m6A-RT-QPCR detects inflammation related m6A methylation changes. To assess the ability of the m6A-RT-QPCR method to observe methylation changes, we evaluated the alterations in m6A methylation of the selected SOCS1 and SOCS3 motifs in different inflammation related scenarios. The mRNA methylation of 

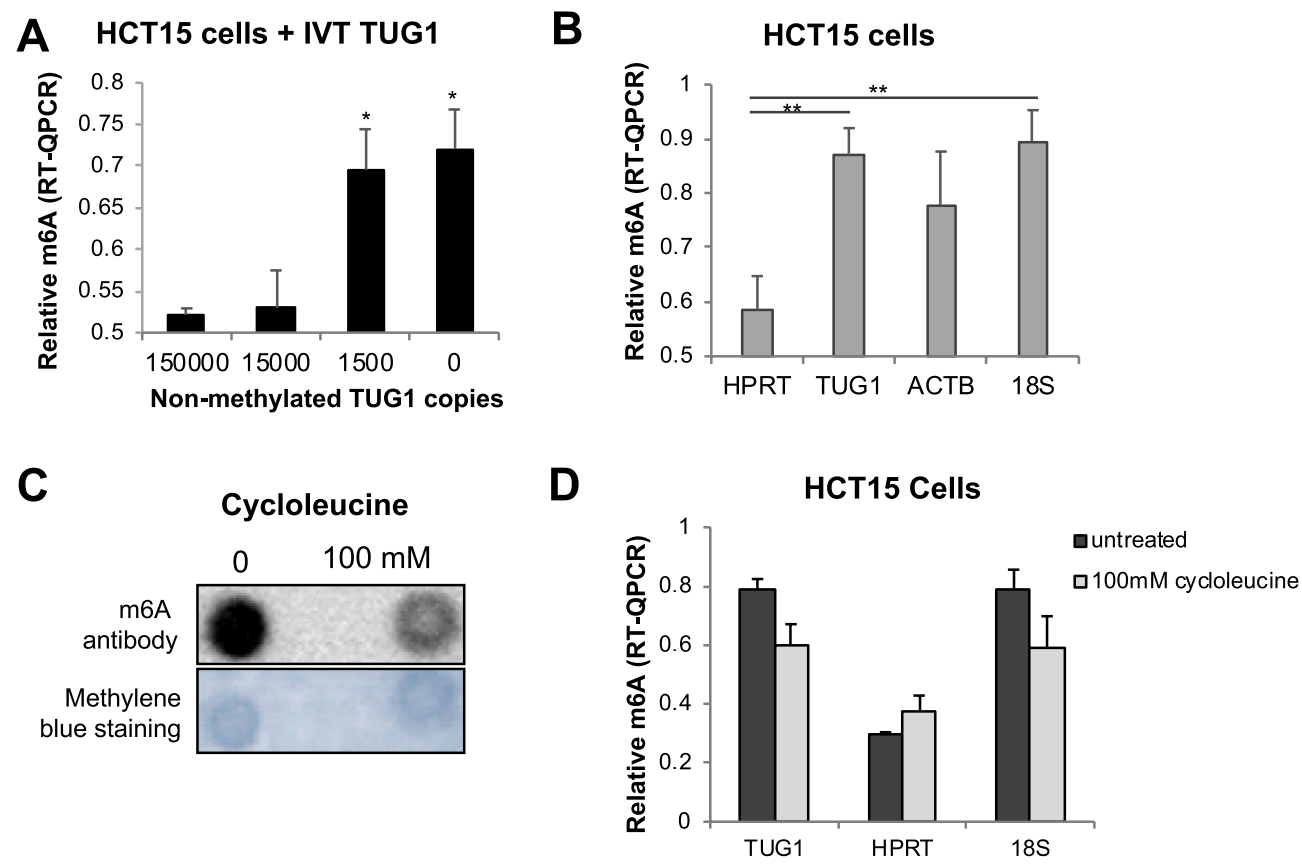

Figure 2. (A) Relative m6A quantification of TUG1 motif in basal RNA (0) and in RNA mixed with increasing amount of copies (1500, 150000 and 1500000) of in vitro transcribed non-methylated RNA. $*$ p-value $<0.05$ based on paired Student's t-test. (B) Relative quantification of previously described methylated motifs using m6A-RT-QPCR method. **p-value $<0.01$ based on paired Student's t-test. (C) HCT15 cells were treated with different concentrations of cycloleucine 3 hours prior to RNA extraction and total RNA was evaluated for the quantification of the m6A signal. Representative m6A RNA immuno-dot blot of basal and $100 \mathrm{mM}$ cycloleucine treatment of 4 independent experiments. Methylene blue staining was used as a loading control for RNA amount. (D) Relative quantification of the m6A levels in the selected motifs of TUG1 and $18 \mathrm{~S}$ in RNA extracted from non-treated (-) and $100 \mathrm{mM}$ cycloleucine treated cells. HPRT was used as a negative control. Data are represented as the mean and standard error of four independent experiments.

these genes has been shown to be stimulus-specific in T-cells, where stimulation with IL7 induces m6A methylation and subsequent degradation of these transcripts ${ }^{5}$.

Celiac disease (CeD) and type 1 diabetes (T1D) are chronic inflammatory conditions of the small intestine and the pancreas, respectively. SOCS genes have been involved in the pathogenesis of these diseases, and it is known that celiac and diabetic patients show alterations in these genes at both mRNA and protein levels ${ }^{19,20}$. We used RNA extracted from intestinal biopsy samples of celiac disease patients and non-celiac controls to analyze the levels of m6A methylation in the selected motifs of SOCS genes. Similarly to what happened in the intestinal cell lines, the SOCS3 motif analyzed presented significantly higher m6A levels compared to HPRT. However, SOCS1 levels were only significant in the active disease biopsies (Fig. 4A). The mean methylation of both motifs shows a change in the disease status, with a tendency to higher methylation levels for SOCS1 and lower methylation for SOCS3 motif in the celiac patient group (Fig. 4A). The m6A-RT-QPCR technique was also used to quantify the levels of m6A SOCS gene motifs in in vitro cultured pancreatic $\beta$-cells. In this case, cells were stimulated with IFN $\gamma$ and IL1 $\beta$ to simulate the inflammatory environment characteristic of the $\beta$-cell destruction that occurs in T1D. In contrast to what we had observed in the intestinal biopsies, $ß$-cells treated with the pro-inflammatory cytokines showed no methylation of the SOCS1 motif and a significantly higher methylation of the SOCS3 motif (Fig. 4B).

\section{Discussion}

N6-Methyladenine (m6A) is the most abundant internal modification on mammalian RNAs constituting a new layer of gene regulation that is involved in different biological processes as T-cell response or tumorigenesis ${ }^{5,21}$. Moreover, it has been shown that this modification plays important roles in the development of several disorders, as neuronal disorders, immune-mediated diseases, obesity and cancer ${ }^{2}$. Although several techniques have been developed in order to map and quantify m6A residues ${ }^{9,10,22}$, available methods are laborious or involve next-generation sequencing, so new approaches are needed to measure the m6A levels in selected transcripts that could be involved in the development of disease.

In this study we have taken advantage of the previously described diminished capacity of BstI enzyme to retrotranscribe $\mathrm{m} 6 \mathrm{~A}$ nucleotides located adjacent to the priming oligo ${ }^{11,12}$. Using the method described here we have been able to confirm the methylation motifs described by other techniques in TUG1 lncRNA, ACTB mRNA and $18 S$ rRNA in intestinal cell lines, in a cost-affordable and time-efficient way. Additionally, when comparing the results of specific residues using our method to those of $\mathrm{m} 6 \mathrm{~A}$ immunoprecipitation experiments, where a 
A

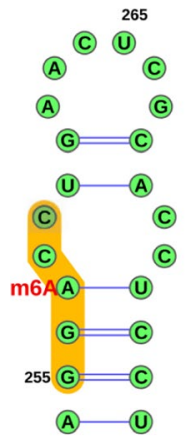

275

B

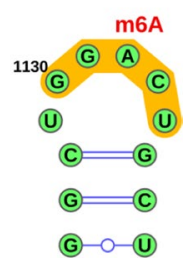

C

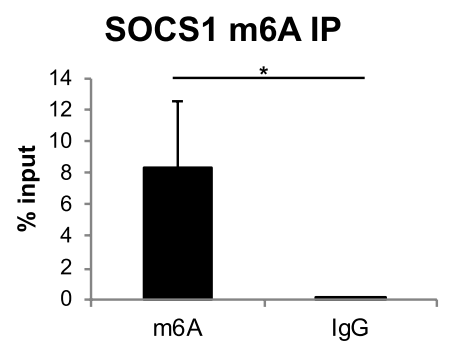

D

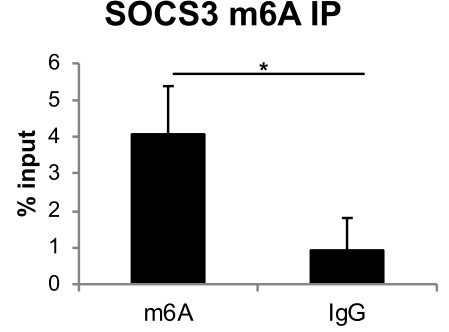

E

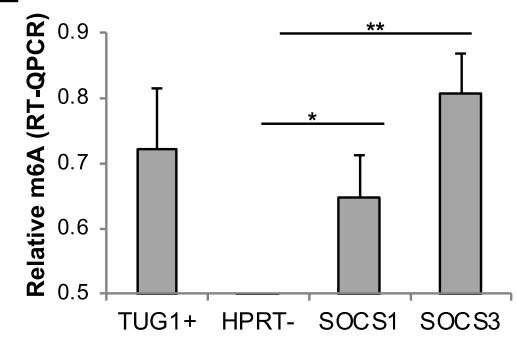

Caco2 cells

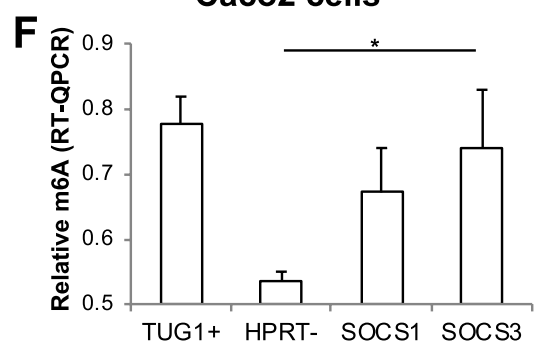

Figure 3. Location of the m6A motifs in the $3^{\prime}$ sites of the (A) SOCS1 and (B) SOCS3 genes. RNA immunoprecipitation of m6A followed by RT-QPCR for the $3^{\prime}$ sites of (C) SOCS1 and (D) SOCS3. Data are represented as the mean and standard error of four independent experiments. p-value $* \mathrm{p}<0.05$. Validation of the m6A-RT-QPCR method for the relative quantification of m6A motifs in SOCS1 and SOCS3 genes in RNA extracted from (E) HCT15 and (F) Caco2 cells. Data are represented as the mean and standard error of three independent experiments. ${ }^{*} \mathrm{p}$-value $<0.01,{ }^{*} \mathrm{p}<0.05$; based on paired Student's t-test.

A

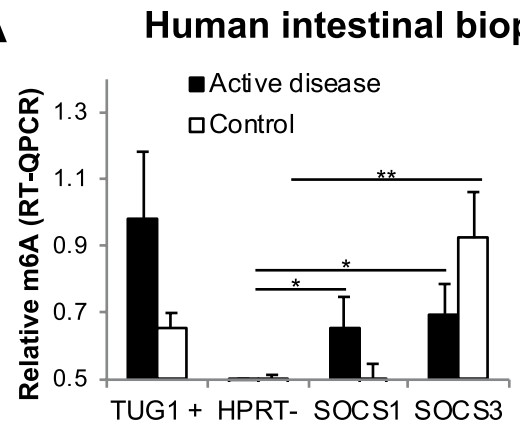

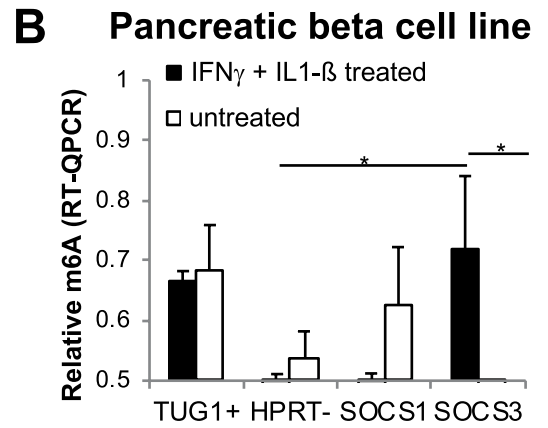

Figure 4. (A) Relative m6A quantification of selected SOCS1 and SOCS3 motifs in intestinal biopsies of celiac patients $(n=8)$ and controls $(n=6)$. Data are represented as the mean and standard error of all samples in each group. ${ }^{*}$ p-value $<0.01 ; * 0.05$ based on unpaired Student's t-test. (B) Relative m6A quantification of selected SOCS 1 and SOCS3 motifs in pancreatic $\beta$-cells stimulated with IFN $\gamma$ and IL1 $\beta$ or without stimulation. Data are represented as the mean and standard error of three independent experiments. *p-value $<0.05$ based on paired Student's t-test.

region is evaluated, we found that the methylation status in concordant in eight out of ten of the RNAs further supporting the validity of the method.

This study shows that this method is useful for the relative quantification of a specific residue within a transcript. When a non-methylated RNA is added to basal RNA, decreasing the percentage of m6A, or cycloleucine is used to reduce the overall RNA methylation levels in the cell, values obtained using m6A-RT-QPCR decreased, confirming the validity of the technique to evaluate if a certain residue presents alterations in its methylation rates in response to different stimuli or disease status. Although the experiments performed using IVT RNAs suggests that $\mathrm{m} 6 \mathrm{~A}$ percentages as low as $1 \%$, resembling what has been described for SCARLET ${ }^{9}$, could be detected using this technique, the exact percentages are not possible to determine due to the extreme difficulty to artificially synthesize a stable RNA with the same characteristics as the regions that are being analyzed (i.e $140 \mathrm{bp}$ ).

This technique can be used with limited amounts of RNA extracted from human tissues, that normally are not enough to perform an RNA immunoprecipitation, and the relative amount of m6A marks in candidate regions can be easily quantified. We have demonstrated the usefulness of this approach for the quantification of residue 
specific methylation in the $3^{\prime}$ UTR of SOCS1 and SOCS3 transcripts that were recently described to be regulated by m6A modifications in the context of immune response ${ }^{5}$. Moreover, using this technique, we have shown that there are inflammation-related changes in the mRNA methylation of specific motifs of these two genes. It has been known for a long time that SOCS proteins play an important part in the fine tuning of the immune response and inflammation ${ }^{16}$ being involved in inflammatory diseases as CeD or T1D, the inflammation-related variability of the m6A methylation in these transcripts, and other interesting candidates, could provide progress on the understanding on their contribution in disease pathogenesis.

\section{Material and Methods}

Biopsies and cell lines. Celiac disease in pediatric patients was diagnosed according to the ESPGHAN (European Society of Pediatric Gastroenterology Hematology and Nutrition) criteria in force at the time of recruitment, including anti-gliadin (AGA), antiendomysium (EMA) and anti-transglutaminase antibody (TGA) determinations as well as a confirmatory small bowel biopsy. The study was approved by the Cruces University Hospital Ethics Board and analyses were performed after informed consent was obtained from all subjects or their parents. All experiments were performed in accordance with relevant guidelines and regulations. Biopsy specimens from the distal duodenum of each patient were obtained during routine diagnosis endoscopy. None of the patients suffered from any other concomitant immunological disease. None of the controls showed small intestinal inflammation at the time of the biopsy.

Intestinal Caco2 (86010202) and HCT15 (91030712) cell lines were purchased from Sigma Aldrich. HCT15 cells were incubated with $100 \mathrm{mM}$ cycloleucine (Sigma Aldrich) for 3 hours prior to RNA extraction. The EndoC- $\beta$ H1 human $\beta$-cell line (provided by Dr. R. Scharfmann, Centre de Recherche de l'Institut du Cerveau et de la Moelle Épinière, Paris, France) was cultured in plates coated with Matrigel-fibronectin $(100 \mathrm{mg} / \mathrm{ml}$ and $2 \mathrm{mg} / \mathrm{mL}$, respectively) in low-glucose DMEM as previously described ${ }^{23}$. Human pancreatic ${ }^{\circledR}$-cells were exposed to IL-1ß (R\&D Systems, Abingdon,U.K.) at 50 units/mL and IFN $\gamma$ (PeproTech, Rocky Hill, NJ) at 1,000 units/mL for $24 \mathrm{~h}$.

RNA, retrotranscription, PCR and QPCR. Total RNA was extracted from cells and biopsies using QIAGEN RNA mini kit. All samples were subjected to DNAse I treatment during extraction.

For the m6A-retrotranscription reaction, 75-150 ng of RNA, $100 \mathrm{nM}$ of each primer, 50 (M dNTPs and $0.1 \mathrm{U}$ of $B s t I$ (NEB) or $0.8 \mathrm{U}$ of $M R T$ (ThermoScientific) were used. The cycling conditions were as follows: $50^{\circ} \mathrm{C}$ $-5 / 15 / 30 \mathrm{~min}, 85^{\circ} \mathrm{C}-3 \mathrm{~min}, 4^{\circ}-\infty$. For the PCR, 1 (1 of the RT reaction was amplified using Dream Taq polymerase (ThermoScientific) and $100 \mathrm{nM}$ of each primer. Cycling conditions were as follows: $95^{\circ} \mathrm{C}-3 \mathrm{~min}, 40 \mathrm{cycles} \mathrm{x}$ $\left(95^{\circ} \mathrm{C}-30 \mathrm{sg}, 60^{\circ} \mathrm{C}-30 \mathrm{sg}, 72^{\circ} \mathrm{C}-30 \mathrm{sg}\right), 4^{\circ} \mathrm{C}-\infty$. For the QCPR, 1.5 (l of the retrotranscription reaction was used together with $100 \mathrm{nM}$ of each primer and 2X iTaq SYBR green (BioRad). Reactions were run in an Illumina Eco Real Time System and melting curves were analyzed to ensure the amplification of a single product. The sequences of all the primers are available upon request.

In vitro transcription (IVT). TUG1 amplicon was reamplified using a forward primer with a T7 sequence. After purification, $100 \mathrm{ng}$ of DNA were used for in vitro transcription using T7 polymerase and rNTPs from Takara (Clonthech) following manufacturer's instructions. The IVT product was purified using the Direct-zol miniprep kit (Zymo Research).

Dot blot. For cycloleucine experiments $400 \mathrm{ng}$ of RNA were crosslinked into a nitrocellulose membrane using UV. Membrane was blocked using 5\% milk in $0.1 \%$ PBST ( $0.1 \%$ Tween in PBS). Membrane was incubated overnight with rabbit m6A antibody (1:500) (Abcam, ab151230) at $4{ }^{\circ} \mathrm{C}$ followed by an incubation with a secondary HRP anti-rabbit antibody. Membrane was developed using SuperSignal West Femto substrate (Thermofisher) in a ChemiDoc gel imaging system (Biorad).

m6A RNA immunoprecipitation. $2 \mu \mathrm{g}$ of RNA per sample were fragmented with RNA fragmentation buffer $\left(100 \mathrm{mM}\right.$ Tris, $\left.2 \mathrm{mM} \mathrm{MgCl}_{2}\right)$ for $3 \mathrm{~min}$ at $95^{\circ} \mathrm{C}$ and placed on ice immediately after heating. $10 \%$ of RNA was kept as input. $1 \mu \mathrm{g}$ of $\mathrm{m} 6 \mathrm{~A}$ antibody (Abcam, ab151230) was coupled to agarose A beads (GE Healthcare) in a rotation wheel for $1 \mathrm{~h}$ at $4^{\circ} \mathrm{C}$. After incubation, beads were washed twice in reaction buffer $(150 \mathrm{mM} \mathrm{NaCl}, 10 \mathrm{mM}$ Tris- $\mathrm{HCl}, 0.1 \% \mathrm{NP}-40)$, RNA was added to the antibody coupled beads and incubated for $3 \mathrm{~h}$ at $4{ }^{\circ} \mathrm{C}$ in a rotating wheel. Subsequently, beads were washed $2 \mathrm{X}$ in reaction buffer, $2 \mathrm{X}$ in low salt buffer $(50 \mathrm{mM} \mathrm{NaCl}, 10 \mathrm{mM}$ TrisHCl and $0.1 \% \mathrm{NP}-40)$ and $2 \mathrm{X}$ in high salt buffer $(500 \mathrm{mM} \mathrm{NaCl}, 10 \mathrm{mM}$ TrisHCl and $0.1 \% \mathrm{NP}-40)$. After the last wash, beads were resuspended in Lysis buffer and RNA was extracted using the PureLink RNA extraction kit (Ambion).

Statistical analysis. All experiments were performed in triplicate. The data are represented as the mean \pm standard error of the mean. Comparisons were analyzed by Student's $t$ test. The significance level was set at $\mathrm{p}<0.05$.

\section{References}

1. Fu, Y., Dominissini, D., Rechavi, G. \& He, C. Gene expression regulation mediated through reversible m(6)A RNA methylation. Nat Rev Genet. 15, 293-306 (2014).

2. Maity, A. \& Das, B. N6-methyladenosine modification in mRNA: machinery, function and implications for health and diseases. FEBS J. 283, 1607-1630 (2016).

3. Yue, Y., Liu, J. \& He, C. RNA N6-methyladenosine methylation in post-transcriptional gene expression regulation. Genes Dev. 29(13), 1343-1355 (2015).

4. Cao, G., Li, H. B., Yin, Z. \& Flavell, R. A. Recent advances in dynamic m6A RNA modification. Open Biol. 6(4), 160003, https://doi. org/10.1098/rsob.160003 (2016). 
5. Li, H. B. et al. m6A mRNA methylation controls T cell homeostasis by targeting the IL-7/STAT5/SOCS pathways. Nature. 548, 338-342 (2017).

6. Tong, J. et al. m6A mRNA methylation sustains Treg suprressive functions. Cell Research. 28(2), 253-256 (2018).

7. Feng, Z., Li, Q., Meng, R., Yi, B. \& Xu, Q. METTL3 regulates alternative splicing of MyD88 upon lipopolysachcaride-induced inflammatory response in human dental pulp cells. J. Cell. Mol. Med. 10(19), 1-11 (2018).

8. Roignant, J. Y. \& Soller, M. m6A in mRNA: An Ancient Mechanism for Fine-Tuning Gene Expression. Trends in Genetics. 33(6), 380-390 (2017)

9. Liu, N. et al. Probing N6-methyladenosine RNA modification status at single nucleotide resolution in mRNA and long noncoding RNA. RNA. 19(12), 1848-1856 (2013).

10. Linder, B. et al. Single-nucleotide-resolution mapping of $\mathrm{m} 6 \mathrm{~A}$ and $\mathrm{m} 6 \mathrm{Am}$ throughout the transcriptome. Nature Methods. 12(8), 767-72 (2015)

11. Harcourt, E. M., Ehrenschwender, T., Batista, P. J., Chang, H. Y. \& Kool, E. T. Identification of a Selective Polymerase Enables Detection of N6-Methyladenosine in RNA. J. Am. Chem. Soc. 135(51), 19079-19082 (2013).

12. Wang, S. et al. N6-Methyladenine hinders RNA- and DNA-directed DNA synthesis: application in human rRNA methylation analysis of clinical specimens. Chem Sci. 7, 1440-1446 (2016).

13. Kiefer, J. R., Braman, J. C. \& Beese, L. S. Visualizing DNA replication in a catalytically active Bacillus DNA polymerase crystal. Nature. 391, 304-307 (1998).

14. Zhou, J. et al. Dynamic m6A mRNA methylation directs translational control of heat shock response. Nature. 526(7574), 591-594 (2015).

15. Shi, C., Shen, X., Niu, S. \& Ma, C. Innate Reverse Transcriptase Activity of DNA Polymerase for Isothermal RNA Direct Detection. J. Am. Chem. Soc. 137(43), 13804-13806 (2015).

16. Yoshimura, A., Suzuki, M., Sakaguchi, R., Hanada, T. \& Yasukawa, H. SOCS, Inflammation, and Autoimmunity. Frontiers in Immunology. 3, 20, https://doi.org/10.3389/fimmu.2012.00020 (2012).

17. Liu, H. et al. MeT-DB V2.0: elucidating context-specific functions of N6-methyl-adenosine methyltranscriptome. Nucleic Acid Research. 46(D1), 281-287 (2018).

18. Finkel, D. \& Groner, Y. Metylations of adenosine residues (m6A) in pre-mRNA are important for formation of late simian virus 40 mRNAs. Virology. 131(2), 409-425 (1983).

19. Mazzarella, G. et al. Constitutive activation of the signal transducer and activator of transcription pathway in celiac disease lesions. Am J Pathol. 162(6), 1845-55 (2003).

20. Liang, Y., Xu, W. D., Peng, H., Pan, H. F. \& Ye, D. Q. SOCS signaling in autoimmune diseases: molecular mechanisms and therapeutic implications. Eur J Immunol. 44(5), 1265-75 (2014).

21. Cui, Q. et al. m6A RNA methylation regulates self-renewal and tumorigenesis of glioblastoma stem cells. Cell Rep. 18(11), 2622-2634 (2017).

22. Aschenbrenner, J., Werner, S. \& Marchand, V. Engineering of a DNA polymerase for direct m6A sequencing. Angew Chem Int Ed Engl. 57(2), 417-421 (2018).

23. Ravassard, P. et al. A genetically engineered human pancreatic $\beta$ cell line exhibiting glucose-inducible insulin secretion. J Clin Invest. 121(9), 3589-97 (2011).

\section{Acknowledgements}

J.R.B. is funded by Project ISCIII-PI16/00258 and co-funded by the European Union ERDF/ESF "A way to make Europe". I.S. is funded by a research project grant 2015111068 of the Basque Department of Health. A.C.R. was funded by a Juan de la Cierva reincorporation and an Ikerbasque fellowships and a research project grant from Asociación Celiacos Madrid. I.R.G., A.O.G. and A.J.M. are supported by predoctoral fellowship grants from the $\mathrm{UPV} / \mathrm{EHU}$ and the Basque Department of Education.

\section{Author Contributions}

A.C.R., I.S., I.R.G., A.O.G. and A.J.M. performed the experiments. M.L. recruited the patients and collected the human samples. A.C.R. and J.R.B. wrote the main manuscript text. All authors reviewed the manuscript.

\section{Additional Information}

Competing Interests: The authors declare no competing interests.

Publisher's note: Springer Nature remains neutral with regard to jurisdictional claims in published maps and institutional affiliations.

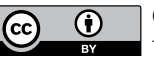

Open Access This article is licensed under a Creative Commons Attribution 4.0 International License, which permits use, sharing, adaptation, distribution and reproduction in any medium or format, as long as you give appropriate credit to the original author(s) and the source, provide a link to the Creative Commons license, and indicate if changes were made. The images or other third party material in this article are included in the article's Creative Commons license, unless indicated otherwise in a credit line to the material. If material is not included in the article's Creative Commons license and your intended use is not permitted by statutory regulation or exceeds the permitted use, you will need to obtain permission directly from the copyright holder. To view a copy of this license, visit http://creativecommons.org/licenses/by/4.0/.

(C) The Author(s) 2019 